\title{
A novel score to predict shunt dependency after aneurysmal subarachnoid hemorrhage
}

\author{
Dominik Diesing, MD, ${ }^{1}$ Stefan Wolf, MD, ${ }^{1}$ Jenny Sommerfeld, ${ }^{1}$ Asita Sarrafzadeh, MD, ${ }^{2}$ \\ Peter Vajkoczy, MD, ${ }^{1}$ and Nora F. Dengler, MD¹
}

\begin{abstract}
1'Department of Neurosurgery, Charité Universitätsmedizin Berlin; and 2Department of Neurosurgery, Universitätsklinikum Heidelberg, Germany
\end{abstract}

\begin{abstract}
OBJECTIVE Feasible clinical scores for predicting shunt-dependent hydrocephalus (SDHC) after aneurysmal subarachnoid hemorrhage (aSAH) are scarce. The chronic hydrocephalus ensuing from SAH score (CHESS) was introduced in 2015 and has a high predictive value for SDHC. Although this score is easy to calculate, several early clinical and radiological factors are required. The authors designed the retrospective analysis described here for external CHESS validation and determination of predictive values for the radiographic Barrow Neurological Institute (BNI) scoring system and a new simplified combined scoring system.
\end{abstract}

METHODS Consecutive data of 314 patients with aSAH were retrospectively analyzed with respect to CHESS parameters and BNI score. A new score, the shunt dependency in aSAH (SDASH) score, was calculated from independent risk factors identified with multivariate analysis.

RESULTS Two hundred twenty-five patients survived the initial phase after the hemorrhage, and $27.1 \%$ of these patients developed SDHC. The SDASH score was developed from results of multivariate analysis, which revealed acute hydrocephalus (aHP), a BNI score of $\geq 3$, and a Hunt and Hess $(\mathrm{HH})$ grade of $\geq 4$ to be independent risk factors for SDHC (ORs 5.709 [aHP], 6.804 [BNI], and 4.122 [HH]; p < 0.001). All 3 SDHC scores tested (CHESS, BNI, and SDASH) reliably predicted chronic hydrocephalus (ORs 1.533 [CHESS], 2.021 [BNI], and 2.496 [SDASH]; $p \leq 0.001$ ). Areas under the receiver operating curve (AUROC) for CHESS and SDASH were comparable (0.769 vs 0.785 , respectively; $p=0.447)$, but the CHESS and SDASH scores were superior to the BNI grading system for predicting SDHC (BNI AUROC 0.649; $p$ $=0.014$ and 0.001 , respectively). In contrast to CHESS and BNI scores, an increase in the SDASH score coincided with a monotonous increase in the risk of developing SDHC.

CONCLUSIONS The newly developed SDASH score is a reliable tool for predicting SDHC. It contains fewer factors and is more intuitive than existing scores that were shown to predict SDHC. A prospective score evaluation is needed.

https://thejns.org/doi/abs/10.3171/2016.12.JNS162400

KEY WORDS shunt-dependent hydrocephalus; aneurysmal subarachnoid hemorrhage; patient outcome; BNI scale; CHESS; SDASH; risk factors; vascular disorders

$\mathrm{S}$ HUNT-dependent hydrocephalus (SDHC) is a common condition after aneurysmal subarachnoid hemorrhage (aSAH). Data indicate that occurrence rates vary between $6.5 \%$ and $67 \%$, and diverse information about potential risk factors exists., ${ }^{4,10,17,21,23,32}$ Early and reliable identification of patients in need of shunt placement might help to decrease the incidence of catheter-related meningitis, shorten the overall hospital stay, and potentially reduce treatment costs. ${ }^{14}$

A recent meta-analysis identified the following risk factors (in decreasing importance): high Fisher grade, acute hydrocephalus (aHP), intracerebral hemorrhage (ICH), intraventricular hemorrhage (IVH), high Hunt and Hess grade, rehemorrhage, posterior circulation location of the

ABBREVIATIONS aHP = acute hydrocephalus; $\mathrm{aSAH}=$ aneurysmal $\mathrm{SAH} ; \mathrm{AUROC}=$ area under the receiver operating curve; $\mathrm{BCl}=$ bicaudate index; $\mathrm{BNI}=\mathrm{Barrow} \mathrm{Neu}-$ rological Institute; $\mathrm{CHESS}=$ chronic hydrocephalus ensuing from SAH score; FRI = failure risk index; ICH = intracerebral hemorrhage; IVH = intraventricular hemorrhage; $\mathrm{SAH}=$ subarachnoid hemorrhage; $\mathrm{SDASH}=$ shunt dependency in $\mathrm{SSAH} ; \mathrm{SDHC}=$ shunt-dependent hydrocephalus .

SUBMITTED September 16, 2016. ACCEPTED December 21, 2016.

INCLUDE WHEN CITING Published online June 9, 2017; DOI: 10.3171/2016.12.JNS162400. 
aneurysm, age $\geq 60$ years, and female sex. ${ }^{28}$ Certain attempts have been made to define scores for predicting the occurrence of SDHC. For example, the failure risk index (FRI) score included the following parameters: third ventricular diameter, Hunt and Hess grade, CSF protein levels, sex, and posterior circulation location of the aneurysm. ${ }^{4}$ So far, the FRI score has not gained wide clinical use. The most recent proposal for such a score is the chronic hydrocephalus ensuing from SAH score (CHESS).${ }^{14}$ Based on a multivariate analysis, the following factors were identified as risks for developing SDHC and therefore were included in the score, which ranges from 1 to 8 points: Hunt and Hess grade $\geq 4$ (1 point), posterior circulation location of the ruptured aneurysm ( 1 point), aHP ( 4 points), the presence of IVH (1 point), and early cerebral infarction found with a follow-up CT scan (1 point). Patients with a CHESS of $\geq 6$ points were at higher risk for SDHC $(p<0.0001)$ than other patients. ${ }^{14}$ Because of these criteria, the CHESS emphasizes the aHP parameter. ${ }^{2}$

Some studies correlated the amount of cisternal blood and the development of chronic hydrocephalus, but strategies for measuring cisternal blood load varied extensively. ${ }^{5,13}$ Introduced in 2012 by colleagues from the Barrow Neurological Institute (BNI) ${ }^{29}$ the BNI score is an easily applicable method for scoring and quantifying the amount of subarachnoidal blood. The BNI score proved to predict angiographic and symptomatic vasospasm as well as CT-identified infarction and clinical outcome. It results in a score of 1 through 5 in accordance with increasing thickness of the subarachnoid blood clot, measured perpendicular to a cistern or fissure (1, no visible SAH; $2, \leq 5$ $\mathrm{mm} ; 3,10 \mathrm{~mm} ; 4,15 \mathrm{~mm} ; 5,20 \mathrm{~mm})$. The presence of IVH was shown to correlate with an increasing BNI score.? The relationship between BNI scoring and SDHC is currently unknown.

With the variety of possible predisposing factors and scoring attempts made, we questioned whether there might be a less complex variable that is available early in the clinical course and can predict SDHC reliably.

We designed this retrospective study to evaluate the predictive value of the BNI scale for the development of SDHC. Moreover, we aimed to validate the CHESS and compare it with the BNI scale in terms of SDHC prediction. In addition, a question arose: does a more simplified score than CHESS exist and reach similar predictive power?

\section{Methods}

\section{Patient Treatment and Data Collection}

Clinical and radiographic data of 314 consecutive patients who suffered an aSAH between 2009 and 2015 were analyzed retrospectively. This data collection was approved by the local ethics committee (approval EA1/291/14). aSAH confirmed by admission CT scanning or xanthochromic CSF was the inclusion criterion. Patients with a bleeding source other than an intracranial aneurysm and/or traumatic origin of the SAH were excluded. The BNI scale and the CHESS were applied according to the literature. ${ }^{14,29}$ The term "acute hydrocephalus" (aHP) was assessed based on the description given by Jabbarli and colleagues ${ }^{14}$ for the CHESS (according to Bae and colleagues, "aHP was defined as development of ventricle enlargement on the basis of the third ventricle width and periventricular low density on CT scan within 72 hours of the aneurysmal rupture, and as a clinical manifestation such as mental deterioration, memory impairment, gait disturbance, and urinary incontinence"). ${ }^{2}$ Moreover, the bicaudate index $(\mathrm{BCI})^{3,26}$ was measured. Patients with a BCI of $>0.18$ but a discrepancy in qualitative ventricular evaluation were reevaluated by a second reviewer to reduce possible bias of the variable aHP. Radiographic information, consisting of initial admission $\mathrm{CT}$, postintervention $\mathrm{CT}$, and discharge CT or MRI, was evaluated. Scores were applied as described in the literature. ${ }^{11,29}$ A clinical evaluation was performed at admission using the Hunt and Hess grading system. ${ }^{13}$ Our local treatment protocol was applied as previously described. ${ }^{22}$ Briefly, all patients were treated with attempted early aneurysm occlusion within 48 hours after ictus, according to international guidelines. ${ }^{8,24}$ Posthemorrhagic hydrocephalus management was equivalent with the protocol used by Jabbarli and colleagues. ${ }^{16}$ An external ventricular drain or lumbar drain challenge was performed. A ventriculoperitoneal shunt was placed when temporary external ventricular or lumbar drain occlusion after the acute phase of SAH (14 days) resulted in significant patient deterioration or an increase in ventricle width. Information about shunt dependency and clinical outcome was acquired from files during scheduled control visits 6-12 months after the initial hemorrhage and classified using the modified Rankin Scale. ${ }^{26}$ When this follow-up was not available, a telephone interview was performed. For evaluations of shunt dependency, only patients who survived the initial hospital stay were included.

\section{Scores for Assessing SDHC CHESS}

The CHESS was applied as described by Jabbarli and colleagues. ${ }^{14}$ The following parameters were included in the CHESS, which ranges from 1 to 8 points: Hunt and Hess grade $\geq 4$ (1 point), posterior circulation location of the ruptured aneurysm (1 point), aHP (4 points), the presence of IVH (1 point), and early cerebral infarction identified on CT imaging (1 point).

\section{BNI}

The BNI score was assessed as described by Wilson and colleagues. ${ }^{29}$ Scores 1-5 were differentiated according to the thickness of the subarachnoid blood clot measured perpendicular to a cistern or fissure, as described earlier (1: no visible SAH; 2: $5 \mathrm{~mm} ; 3: 6-10 \mathrm{~mm}$; 4 : 11-15 mm; 5: 16-20 mm).

\section{SDASH}

Based on the results of multivariate analysis, our new simple score variant for predicting SDHC, which ranges from 1 to 4 points, was tested in our patient cohort. The following parameters were included: presence of aHP (2 points), BNI score $\geq 3$ (1 point), and Hunt and Hess grade $\geq 4$ (1 point). 


\section{Data Analysis}

For statistical analysis, SPSS 23 (IBM SPSS) and R 3.2.0 (R Software Foundation) were used. All values are expressed as mean and standard deviation (SD) or median and interquartile range and were compared by the nonparametric Mann-Whitney U, Fisher exact, Spearman rho, or Kruskal-Wallis test, as appropriate. Binary logistic regression analysis was performed. Data are given as ORs with 95\% CIs. The 3 different scores were treated as continuous scores for the calculation of ORs. Statistical significance was assumed at a $p$ value of $\leq 0.05$. Areas under the receiver operating curve (AUROCs) were calculated. The Hosmer-Lemeshow test was performed to validate the goodness of fit for logistic regression models. Comparison of AUROC was performed using the DeLong test. ${ }^{6}$

\section{Results}

\section{Patient Characteristics}

Three hundred fourteen patients were treated at a single institution between January 2009 and December 2015. Two hundred twenty-five patients survived the initial phase after the hemorrhage and were at risk for the development of SDHC; $27.1 \%$ (61) of these patients developed SDHC and underwent a shunt-placement procedure. The demographic characteristics and factors with possible relevance for the development of SDHC are listed in Table 1. Patients who developed SDHC had a significantly poorer clinical state and poorer radiographic scores at admission and worse clinical outcome at follow-up (Table 1).

\section{Univariate and Multivariate Risk Factors for the Development of SDHC}

Univariate risk factors for the development of SDHC were assessed. ORs and $\mathrm{p}$ values of all variables tested are listed in Table 2. IVH, age, presence of aHP, Hunt and Hess grade, and radiographic scores, such as BNI score and original Fisher scale, were identified to be risk factors for SDHC. Posterior location of the aneurysm was of no predictive value, nor was the presence of early infarction on CT imaging after initial aneurysm treatment $(\mathrm{p}=0.893$ and 0.397 , respectively) (Table 2). To identify the strongest prediction parameters and rule out possible colinearities, multivariate analysis of significant factors was performed. IVH, age, and Fisher grade lost their predictive capability, whereas the presence of aHP, a Hunt and Hess grade of $\geq$ 4 , and a BNI score of $\geq 3$ were of significant independent predictive value ( $\mathrm{p}=0.002,0.001$, and 0.020 , respectively) (Table 2).

\section{Prediction of SDHC With the CHESS}

A strong correlation between the CHESS and the development of shunt dependency was found: $7.7 \%$ of the patients who were scored at $0 ; 26.7 \%$ who were scored at 1 ; $28.6 \%$ who were scored at $2 ; 75.0 \%$ who were scored at 3 ; $36.4 \%$ who were scored at $4 ; 41.2 \%$ who were scored at 5 ; $73.9 \%$ who were scored at 6 ; and $66.7 \%$ who were scored at 7 developed SDHC $(p<0.001)$. The maximum score of 8 points was not reached by anyone in our patient cohort (Fig. 1A). The OR for the CHESS to predict SDHC, when
TABLE 1. Patient characteristics

\begin{tabular}{|c|c|c|c|}
\hline Characteristic & $\begin{array}{l}\text { Patients } \\
\text { w/o SDHC }\end{array}$ & $\begin{array}{l}\text { Patients } \\
\text { w/ SDHC }\end{array}$ & $\begin{array}{c}p \\
\text { Value }\end{array}$ \\
\hline Total no. & 164 & 61 & \\
\hline Age (median [IQR]) (yrs) & $51(50-54)$ & $56(53-59)$ & 0.705 \\
\hline Sex, female (\% [n]) & $66.5(109)$ & $70.5(43)$ & 0.566 \\
\hline \multicolumn{4}{|l|}{ Hunt \& Hess grade (\% [n]) } \\
\hline 1 & $40.9(67)$ & $11.5(7)$ & \\
\hline 2 & $25.0(41)$ & $18(11)$ & \\
\hline 3 & $17.7(29)$ & $21.3(13)$ & \\
\hline 4 & $6.1(10)$ & $18(11)$ & \\
\hline 5 & $10.4(17)$ & $31.1(19)$ & $\leq 0.001$ \\
\hline \multicolumn{4}{|l|}{ BNI score (\% [n]) } \\
\hline 1 & $10.4(17)$ & $0(0)$ & \\
\hline 2 & $22.0(36)$ & $6.6(4)$ & \\
\hline 3 & $33.5(55)$ & $39.3(24)$ & \\
\hline 4 & $29.3(48)$ & $37.7(23)$ & \\
\hline 5 & $4.9(8)$ & $16.4(10)$ & 0.006 \\
\hline \multicolumn{4}{|l|}{ Original Fisher grade (\% [n]) } \\
\hline 1 & $6.1(10)$ & $1.6(1)$ & \\
\hline 2 & $5.5(9)$ & $1.6(1)$ & \\
\hline 3 & $75(123)$ & $77.0(47)$ & \\
\hline 4 & $13.4(22)$ & $19.7(12)$ & 0.215 \\
\hline \multicolumn{4}{|l|}{ Aneurysm location (\% [n]) } \\
\hline MCA & $28.0(46)$ & $29.5(18)$ & \\
\hline ACA & $36.6(60)$ & $37.7(23)$ & \\
\hline ICA & $20.7(34)$ & $19.7(12)$ & \\
\hline Posterior circulation & $14.6(24)$ & $13.1(8)$ & 0.985 \\
\hline \multicolumn{4}{|l|}{ Intervention (\% [n]) } \\
\hline Clipping & $68.3(112)$ & $59.0(36)$ & \\
\hline Coiling & $28.7(47)$ & $36.1(22)$ & \\
\hline Other & $1.8(3)$ & $4.9(3)$ & \\
\hline None & $1.2(2)$ & $0(0)$ & 0.294 \\
\hline Presence of IVH (\% [n]) & $34.1(56)$ & $73.8(45)$ & $\leq 0.001$ \\
\hline Early ischemia (\% [n]) & $8.0(13)^{*}$ & $9.8(6)$ & 0.640 \\
\hline $\operatorname{aHP}(\%[n])$ & $14.0(23)$ & $52.5(32)$ & $\leq 0.001$ \\
\hline Posterior aneurysm location (\% [n]) & $13.4(22)$ & $14.8(9)$ & 0.893 \\
\hline \multicolumn{4}{|l|}{ Outcome (mRS) $(\%[n]) \dagger$} \\
\hline 0 & $38.9(58)$ & $8.6(5)$ & \\
\hline 1 & $36.2(54)$ & $15.5(9)$ & \\
\hline 2 & $9.4(14)$ & $8.6(5)$ & \\
\hline 3 & $6.7(10)$ & $24.1(14)$ & \\
\hline 4 & $5.4(8)$ & $24.1(14)$ & \\
\hline 5 & $1.3(2)$ & $19(11)$ & \\
\hline 6 & $2(3)$ & $0(0)$ & $\leq 0.001$ \\
\hline Lost to follow-up (n) & 15 & 3 & \\
\hline
\end{tabular}

$\mathrm{ACA}=$ anterior cerebral artery; ICA = internal carotid artery; IQR = interquartile range; $\mathrm{MCA}=$ middle cerebral artery; $\mathrm{mRS}=$ modified Rankin Scale.

Boldface type indicates a significant result.

* Evaluated only in patients who underwent intervention ( $n=162$ in the nonSDHC group).

$\dagger$ Evaluated in patients with follow-up (149 in the non-SDHC group and 58 in the SDHC group). 
TABLE 2. Univariate and multivariate risk factors for SDHC

\begin{tabular}{|c|c|c|c|}
\hline Risk Factors & OR & $95 \% \mathrm{Cl}$ & $p$ Value \\
\hline \multicolumn{4}{|c|}{$\begin{array}{l}\text { Possible relevance for development } \\
\text { of SDHC (univariate analysis) }\end{array}$} \\
\hline IVH & 4.670 & $2.403-9.076$ & $<0.001$ \\
\hline Sex & 1.194 & $0.632-2.258$ & 0.585 \\
\hline Age & 1.036 & $1.006-1.066$ & 0.018 \\
\hline aHP & 5.709 & $2.915-11.181$ & $<0.001$ \\
\hline $\mathrm{HH}$ & 1.822 & $1.461-2.273$ & $<0.001$ \\
\hline $\mathrm{HH}$ grade $\geq 4$ & 4.122 & $2.143-7.927$ & $<0.001$ \\
\hline Posterior circulation & 0.944 & $0.407-2.189$ & 0.893 \\
\hline Early infarction & 2.397 & $0.470-12.224$ & 0.397 \\
\hline BNI score $\geq 3$ & 6.804 & $2.345-19.743$ & $<0.001$ \\
\hline Original Fisher grade & 1.734 & $1.011-2.974$ & 0.045 \\
\hline \multicolumn{4}{|l|}{$\begin{array}{l}\text { Analysis of significant factors } \\
\text { (multivariate analysis) }\end{array}$} \\
\hline IVH & 1.480 & $0.612-3.582$ & 0.385 \\
\hline Age & 1.013 & $0.978-1.050$ & 0.463 \\
\hline aHP & 3.814 & $1.823-7.979$ & 0.002 \\
\hline $\mathrm{HH}$ grade $\geq 4$ & 2.731 & $1.278-5.837$ & 0.001 \\
\hline BNI score $\geq 3$ & 3.873 & $1.233-12.158$ & 0.020 \\
\hline Original Fisher grade & 0.563 & $0.217-1.464$ & 0.239 \\
\hline \multicolumn{4}{|c|}{$\begin{array}{l}\text { Predictive power of BNI, CHESS, \& } \\
\text { SDASH scores }\end{array}$} \\
\hline $\mathrm{BNI}$ & 2.021 & $1.447-2.822$ & $<0.001$ \\
\hline CHESS & 1.533 & $1.325-1.775$ & $<0.001$ \\
\hline SDASH & 2.496 & $1.859-3.352$ & $<0.001$ \\
\hline
\end{tabular}

Boldface type indicates a significant result.

analyzed as a continuous score, was 1.533 (CI 1.325-1.775; $\mathrm{p} \leq 0.001$ ) (Fig. 1A, Table 2). The receiver operating curve is shown in Fig. 1. The area under the curve for CHESS was 0.769 .

\section{Prediction of SDHC With the BNI Score}

The BNI score was tested for its power to predict SDHC. The OR for BNI was 2.021 (CI 1.447-2.822; p < $0.001)$. Increasing BNI scores correlated with an increasing prevalence of SDHC $(0 \%$ of patients with BNI Score 1, $10 \%$ with BNI Score 2, 30.4\% with BNI Score 3, 32.4\% with BNI Score 4, and 55.6\% with BNI Score 5; $\mathrm{p}<0.001$ ) (Fig. 1B, Table 2). The AUROC for BNI score was 0.654 (Fig. 1D).

\section{Prediction of SDHC With the Newly Developed SDASH Score and Comparison With Existing Scores}

With an aim to develop a score that is as simple as possible, we included significant parameters according to the results of our multivariate analysis. Calculation of a score based on weighted ORs resulted in a nonlinear distribution of patients who suffered SDHC and a relatively small AUC of 0.665 . Therefore, we modified the approach by trial and error and tested the following point distribution for a new score that we called "shunt dependency in SAH" (SDASH) score: aHP (2 points if present), Hunt and Hess grade $\geq 4$ ( 1 point), and BNI score $\geq 3$ (1 point). This distribution resulted in a score that ranges from 0 to 4 .

The percentage of patients who developed SDHC increased linearly with higher scores ( $p<0.001) ; 2.9 \%$ of patients with an SDASH score of $0,18.6 \%$ of patients with a score of $1,40.6 \%$ with a score of $2,50 \%$ with a score of 3 , and $76.2 \%$ with a score of 4 developed SDHC (Fig. 1C). The OR for the new score to predict SDHC was 2.496 (CI 1.859-3.352; $p<0.001$ ). The AUROC was 0.785 (Fig. 1D). A comparison of SDASH and CHESS revealed no statistically significant difference in predictive performance of the scores $(p=0.447)$. CHESS and SDASH scores were superior to the BNI score for predicting SDHC ( $p=0.014$ and 0.001 , respectively).

\section{Discussion}

Our data show that the recently introduced CHESS reliably predicted the occurrence of SDHC. Moreover, increasing BNI scores predicted SDHC. Additional independent risk factors for the development of SDHC in patients with aSAH were aHP and a Hunt and Hess grade of $\geq 4$. Based on our multivariate analysis, we developed the SDASH score, which included a simple combination of 3 factors, aHP, Hunt and Hess grade, and BNI score. The AUROC of CHESS and SDASH were comparable. The SDASH score resulted in a more intuitive distribution of values and was easier to calculate than the CHESS. No statistically significant difference in score performances between the CHESS and SDASH was detected.

Although they have some predictive potential, clinical or radiographic scores alone did not result in satisfactory accuracy in predicting SDHC, as shown in our analysis and that of others. In our review of the literature, a variety of conditions were identified risk factors for the development of SDHC. 4,19,20,23,30 Therefore, the development of a score that includes a combination of relevant risk factors seemed reasonable. Complex scores that have not gained wide clinical use exist., ${ }^{4,9}$ The newly developed CHESS, introduced in 2016 by Jabbarli and colleagues, ${ }^{14}$ was an easy and accurate approach predicting SDHC. The accuracy of the score was reproduced in our data set, but some factors lost relevance in our univariate and multivariate analyses. Posterior location of the aneurysm did not show predictive value in our patients, which is consistent with the results of other studies that did not find an association of posterior aneurysm location and SDHC. ${ }^{30}$ However, a recent meta-analysis found it to be significant. ${ }^{28}$ These disparities might be attributed to differences in patient characteristics and local treatment strategies, including type of aneurysm treatment, or variations in considering the necessity to place a shunt after aSAH.,18 The risk factor of early cerebral infarction detected by CT was also not significant in our analysis, which is in contrast to the results of Jabbarli and colleagues. An assumed difference in occurrence rates of early cerebral infarction might explain the varied results. ${ }^{14,15}$ The fact that Fisher grade was a relevant predictor for SDHC in our series is consistent with the results of a previous meta-analysis ${ }^{28}$ but does not match the findings of another recent study. ${ }^{14}$ The discrepancy is explained partially by the different scoring 

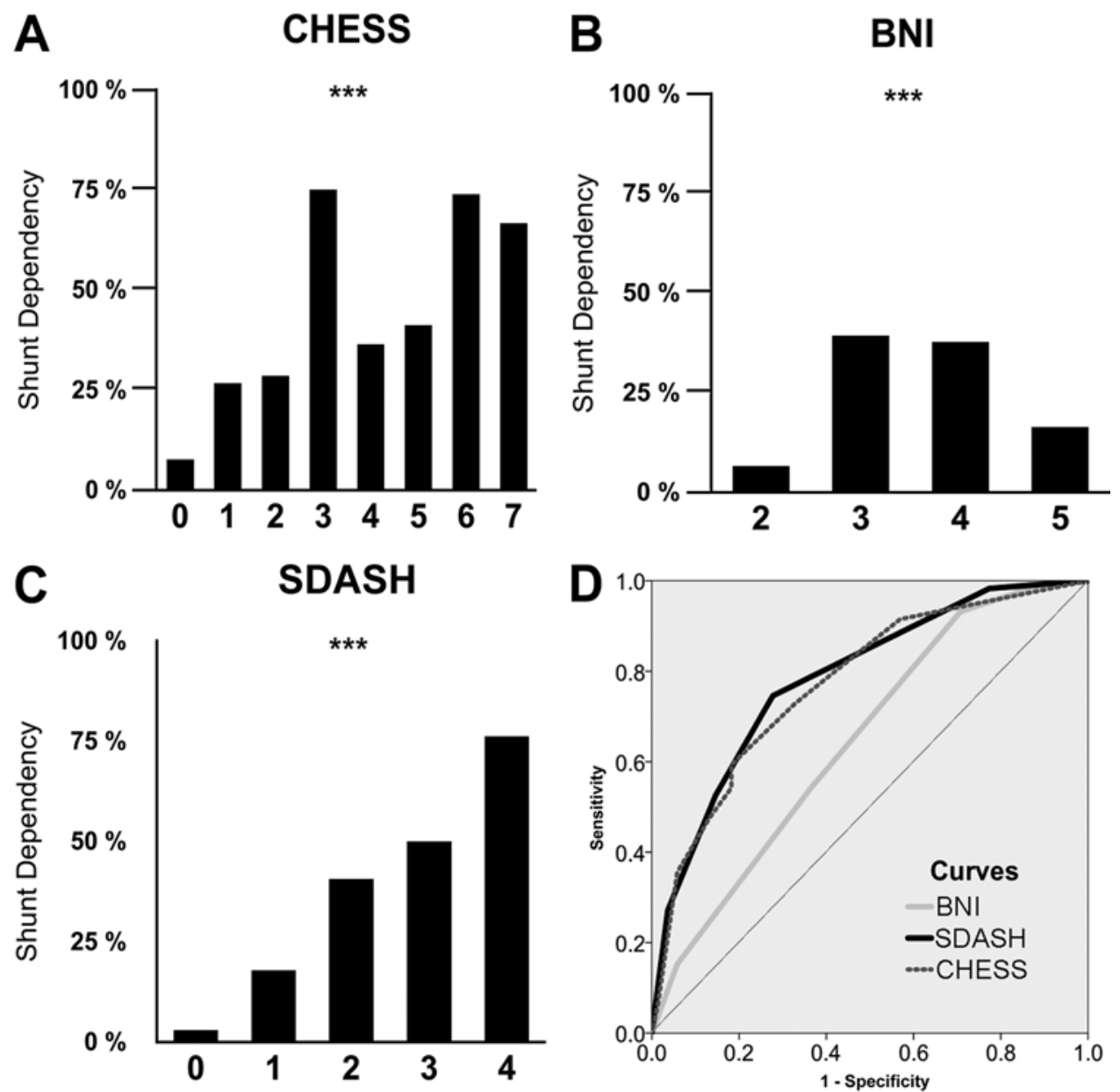

FIG. 1. Development of SDHC in patients with aSAH according to different scores. A: CHESS. B: BNI. C: SDASH. ${ }^{* * *} p \leq 0.001$. D: AUROC.

systems used (modified vs original Fisher scale) with differences in weighting for the presence of IVH and ICH. For the first time, increasing BNI scores were shown to be associated with increasing risk for the development of SDHC. Findings from a previous study in which an association between subarachnoid blood volume and SDHC was assumed support our findings. ${ }^{10}$

Calculation of a score taking the exact weight of multivariate ORs into account did not result in an intuitive, linearly increasing and powerful score with respect to SDHC prediction. However, modifying the weight of the 3 independently predictive parameters revealed a more powerful score with an intuitive distribution of shunt rates. The SDASH provides an easy grading system from 0 to 4 and includes only 3 parameters (aHP, BNI score $\geq 3$, and Hunt and Hess grade $\geq 4$ ). Its accuracy was comparable to that of CHESS, even though CHESS is more complex because more parameters are taken into account. Because of a correlation between BNI score and the presence of IVH, the BNI scale might serve as a variable that describes not only subarachnoidal blood clot load but also IVH. Therefore, it might be an excellent factor for inclusion in a score to predict SDHC that might be influenced by both entities. Use of the SDASH score is somewhat intuitive and resulted in a monotonous increase in the number of predicted patients who required a shunt per increase in score level. In contrast, the CHESS and BNI showed inconsistent frequencies with a decrease in SDHC rates but higher score values at certain points. Another advantage of the SDASH is its calculation from factors that are available at admission and that do not vary during the clinical course or depend on treatment strategies. In the literature, additional but variable factors that influence the development of SDHC, such as the presence of a ventricular drain, increased daily CSF output, and/or aneurysm treatment strategy, have been discussed..$^{1,10,14}$

\section{Limitations}

Its single-center retrospective design reduces the generalizability of our prediction model. An external and prospective evaluation is needed. Other factors, such as the presence of a ventricular drain and the amount of daily CSF output, which might be additional risk factors for shunt placement, ${ }^{25}$ were not included in our analysis. Potential collinearities of these factors with the presence of aHP or severity of SAH exist. Moreover, information about in-hospital complications and recurrent hemorrhage 
was not included. To acknowledge possible difficulties in retrospectively defining aHP, a 2 -sided analysis was performed. One reviewer qualitatively evaluated $\mathrm{CT}$ results at admission according to the definition given by Bae and colleagues ("development of ventricle enlargement on the basis of the third ventricle width and periventricular low density on CT scan within 72 hours of the aneurysmal rupture"). A second evaluation was performed according to BCI diameters. In case of differences, a case-specific review by an additional reviewer was performed until consensus was reached. The definition of chronic hydrocephalus that necessitates a shunt-placement procedure might vary between clinicians, even though a ventricular or lumbar drain challenge was performed. ${ }^{4,14}$ Our usual management after the acute phase included close cooperation with rehabilitation caregivers and readmission to our hospital if routine follow-up CT performed 2 weeks after discharge revealed increasing ventricular width or if clinical deterioration occurred. However, there might be a limited number of patients who developed untreated hydrocephalus that was not recognized this way.

\section{Conclusions}

The newly developed SDASH score is a reliable tool for predicting SDHC. It involves fewer factors and is more intuitive than existing scores that were shown to predict SDHC. A prospective score evaluation is needed.

\section{Acknowledgments}

We thank Norbert Baro for patient management and assistance.

\section{References}

1. Arroyo-Palacios J, Rudz M, Fidler R, Smith W, Ko N, Park S, et al: Characterization of shape differences among ICP pulses predicts outcome of external ventricular drainage weaning trial. Neurocrit Care 25:424-433, 2016

2. Bae IS, Yi HJ, Choi KS, Chun HJ: Comparison of incidence and risk factors for shunt-dependent hydrocephalus in aneurysmal subarachnoid hemorrhage patients. J Cerebrovasc Endovasc Neurosurg 16:78-84, 2014

3. Barr AN, Heinze WJ, Dobben GD, Valvassori GE, Sugar O: Bicaudate index in computerized tomography of Huntington disease and cerebral atrophy. Neurology 28:1196, 1978

4. Chan M, Alaraj A, Calderon M, Herrera SR, Gao W, Ruland $\mathrm{S}$, et al: Prediction of ventriculoperitoneal shunt dependency in patients with aneurysmal subarachnoid hemorrhage. J Neurosurg 110:44-49, 2009

5. Czorlich P, Ricklefs F, Reitz M, Vettorazzi E, Abboud T, Regelsberger J, et al: Impact of intraventricular hemorrhage measured by Graeb and LeRoux score on case fatality risk and chronic hydrocephalus in aneurysmal subarachnoid hemorrhage. Acta Neurochir (Wien) 157:409-415, 2015

6. DeLong ER, DeLong DM, Clarke-Pearson DL: Comparing the areas under two or more correlated receiver operating characteristic curves: a nonparametric approach. Biometrics 44:837-845, 1988

7. Dengler NF, Diesing D, Sarrafzadeh A, Wolf S, Vajkoczy P: The BNI Scale revisited: predictive capabilities for cerebral infarction and clinical outcome in patients with aneurysmal subarachnoid hemorrhage. Neurosurgery [epub ahead of print], 2017

8. Diringer MN, Bleck TP, Claude Hemphill J III, Menon D, Shutter L, Vespa P, et al: Critical care management of patients following aneurysmal subarachnoid hemorrhage: recommendations from the Neurocritical Care Society's Multidisciplinary Consensus Conference. Neurocrit Care 15:211-240, 2011

9. Dorai Z, Hynan LS, Kopitnik TA, Samson D: Factors related to hydrocephalus after aneurysmal subarachnoid hemorrhage. Neurosurgery 52:763-771, 2003

10. Erixon HO, Sorteberg A, Sorteberg W, Eide PK: Predictors of shunt dependency after aneurysmal subarachnoid hemorrhage: results of a single-center clinical trial. Acta Neurochir (Wien) 156:2059-2069, 2014

11. Fisher CM, Kistler JP, Davis JM: Relation of cerebral vasospasm to subarachnoid hemorrhage visualized by computerized tomographic scanning. Neurosurgery 6:1-9, 1980

12. Hasan D, Tanghe HL: Distribution of cisternal blood in patients with acute hydrocephalus after subarachnoid hemorrhage. Ann Neurol 31:374-378, 1992

13. Hunt WE, Hess RM: Surgical risk as related to time of intervention in the repair of intracranial aneurysms. J Neurosurg 28:14-20, 1968

14. Jabbarli R, Bohrer AM, Pierscianek D, Müller D, Wrede KH, Dammann P, et al: The CHESS score: a simple tool for early prediction of shunt dependency after aneurysmal subarachnoid hemorrhage. Eur J Neurol 23:912-918, 2016

15. Jabbarli R, Reinhard M, Niesen WD, Roelz R, Shah M, Kaier $\mathrm{K}$, et al: Predictors and impact of early cerebral infarction after aneurysmal subarachnoid hemorrhage. Eur J Neurol 22:941-947, 2015

16. Jabbarli R, Reinhard M, Roelz R, Shah M, Niesen WD, Kaier $\mathrm{K}$, et al: The predictors and clinical impact of intraventricular hemorrhage in patients with aneurysmal subarachnoid hemorrhage. Int J Stroke 11:68-76, 2016

17. Kim SH, Chung PW, Won YS, Kwon YJ, Shin HC, Choi CS: Effect of cisternal drainage on the shunt dependency following aneurysmal subarachnoid hemorrhage. J Korean Neurosurg Soc 52:441-446, 2012

18. Klopfenstein JD, Kim LJ, Feiz-Erfan I, Hott JS, Goslar P, Zabramski JM, et al: Comparison of rapid and gradual weaning from external ventricular drainage in patients with aneurysmal subarachnoid hemorrhage: a prospective randomized trial. J Neurosurg 100:225-229, 2004

19. Kwon JH, Sung SK, Song YJ, Choi HJ, Huh JT, Kim HD: Predisposing factors related to shunt-dependent chronic hydrocephalus after aneurysmal subarachnoid hemorrhage. J Korean Neurosurg Soc 43:177-181, 2008

20. Lai L, Morgan MK: Predictors of in-hospital shunt-dependent hydrocephalus following rupture of cerebral aneurysms. J Clin Neurosci 20:1134-1138, 2013

21. O'Kelly CJ, Kulkarni AV, Austin PC, Urbach D, Wallace MC: Shunt-dependent hydrocephalus after aneurysmal subarachnoid hemorrhage: incidence, predictors, and revision rates. Clinical article. J Neurosurg 111:1029-1035, 2009

22. Sandow N, Diesing D, Sarrafzadeh A, Vajkoczy P, Wolf S: nimodipine dose reductions in the treatment of patients with aneurysmal subarachnoid hemorrhage. Neurocrit Care 25:29-39, 2016

23. Schmieder K, Koch R, Lücke S, Harders A: Factors influencing shunt dependency after aneurysmal subarachnoid haemorrhage. Zentralbl Neurochir 60:133-140, 1999

24. Steiner T, Juvela S, Unterberg A, Jung C, Forsting M, Rinkel G: European Stroke Organization guidelines for the management of intracranial aneurysms and subarachnoid haemorrhage. Cerebrovasc Dis 35:93-112, 2013

25. Tso MK, Ibrahim GM, Macdonald RL: Predictors of shuntdependent hydrocephalus following aneurysmal subarachnoid hemorrhage. World Neurosurg 86:226-232, 2016

26. van Gijn J, Hijdra A, Wijdicks EF, Vermeulen M, van Crevel $\mathrm{H}$ : Acute hydrocephalus after aneurysmal subarachnoid hemorrhage. J Neurosurg 63:355-362, 1985 
27. van Swieten JC, Koudstaal PJ, Visser MC, Schouten HJ, van Gijn J: Interobserver agreement for the assessment of handicap in stroke patients. Stroke 19:604-607, 1988

28. Wilson CD, Safavi-Abbasi S, Sun H, Kalani MY, Zhao YD, Levitt MR, et al: Meta-analysis and systematic review of risk factors for shunt dependency after aneurysmal subarachnoid hemorrhage. J Neurosurg 126:586-595, 2017

29. Wilson DA, Nakaji P, Abla AA, Uschold TD, Fusco DJ, Oppenlander ME, et al: A simple and quantitative method to predict symptomatic vasospasm after subarachnoid hemorrhage based on computed tomography: beyond the Fisher scale. Neurosurgery 71:869-875, 2012

30. Yamada S, Ishikawa M, Yamamoto K, Ino T, Kimura T, Kobayashi S: Aneurysm location and clipping versus coiling for development of secondary normal-pressure hydrocephalus after aneurysmal subarachnoid hemorrhage: Japanese Stroke DataBank. J Neurosurg 123:1555-1561, 2015

31. Yang TC, Chang CH, Liu YT, Chen YL, Tu PH, Chen HC: Predictors of shunt-dependent chronic hydrocephalus after aneurysmal subarachnoid haemorrhage. Eur Neurol 69:296303,2013

\section{Disclosures}

The authors report no conflict of interest concerning the materials or methods used in this study or the findings specified in this paper.

\section{Author Contributions}

Conception and design: Dengler, Wolf, Sarrafzadeh. Acquisition of data: Dengler, Diesing, Sommerfeld. Analysis and interpretation of data: Dengler, Diesing, Wolf, Vajkoczy. Drafting the article: Dengler, Diesing, Wolf. Critically revising the article: all authors, Reviewed submitted version of manuscript: Dengler. Approved the final version of the manuscript on behalf of all authors: Dengler. Statistical analysis: Dengler, Wolf. Study supervision: Dengler.

\section{Correspondence}

Nora F. Dengler, Department of Neurosurgery, Charité Universitätsmedizin Berlin, Augustenburger Platz 1, Berlin 13353, Germany.email: nora.dengler@charite.de. 